\title{
A novel cell-selection optimization handover for long-term evolution (LTE) macrocellusing fuzzy TOPSIS
}

\begin{abstract}
To satisfy the demand for higher data rate while maintaining the quality of service, a dense long-term evolution (LTE) cells environment is required. This imposes a big challenge to the network when it comes to performing handover (HO). Cell selection has an important influence on network performance, to achieve seamless handover. Although a successful handover is accomplished, it might be to a wrong cell when the selected cell is not an optimal one in terms of signal quality and bandwidth. This may cause significant interference with other cells, handover failure (HOF), or handover ping-pong (HOPP), consequently degrading the cell throughput. To address this issue, we propose a multiple-criteria decision-making method. In this method, we use an integrated fuzzy technique for order preference by using similarity to ideal solution (TOPSIS) on S-criterion, availability of resource blocks (RBs), and uplink signal-to-interference-plus-noise ratio. The conventional cell selection in LTE is based on S-criterion, which is inadequate since it only relies on downlink signal quality. A novel method called fuzzy multiple-criteria cell selection (FMCCS) is proposed in this paper. FMCCS considers RBs utilization and user equipment uplink condition in addition to Scriterion. System analysis demonstrates that FMCCS managed to reduce handover ping-pong and handover failure significantly. This improvement stems from the highly reliable cellselection technique that leads to increased throughput of the cell with a successful handover. The simulation results show that FMCCS outperforms the conventional and cell selection scheme (CSS) methods.
\end{abstract}

Keyword: Cell selection; Fuzzy TOPSIS; Handover (HO); Throughput; Multiple-criteria decision-making (MCDM) 
